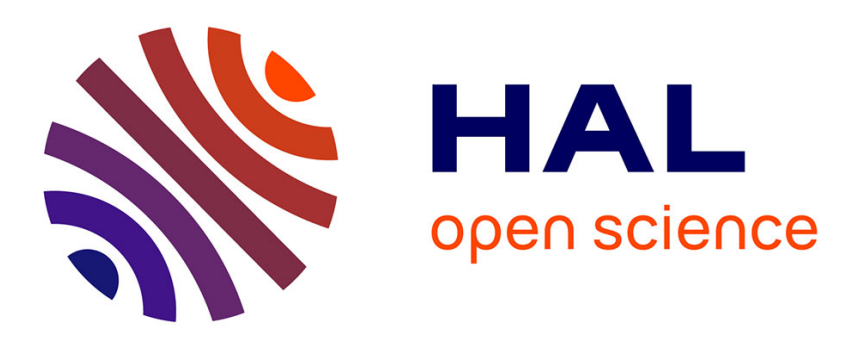

\title{
Polarized Beams Using Scalar Metasurfaces
}

Massimiliano Casaletti, Maciej Smierzchalski, Mauro Ettorre, Ronan Sauleau, Nicolas Capet

\section{To cite this version:}

Massimiliano Casaletti, Maciej Smierzchalski, Mauro Ettorre, Ronan Sauleau, Nicolas Capet. Polarized Beams Using Scalar Metasurfaces. IEEE Transactions on Antennas and Propagation, 2016, 64 (8), pp.3391-3400. 10.1109/TAP.2016.2570251 . hal-01342136

\section{HAL Id: hal-01342136 https://hal.sorbonne-universite.fr/hal-01342136}

Submitted on 30 Jul 2021

HAL is a multi-disciplinary open access archive for the deposit and dissemination of scientific research documents, whether they are published or not. The documents may come from teaching and research institutions in France or abroad, or from public or private research centers.
L'archive ouverte pluridisciplinaire HAL, est destinée au dépôt et à la diffusion de documents scientifiques de niveau recherche, publiés ou non, émanant des établissements d'enseignement et de recherche français ou étrangers, des laboratoires publics ou privés.

\section{(c)(1)}

Distributed under a Creative Commons Attribution| 4.0 International License 


\title{
Polarized Beams Using Scalar Metasurfaces
}

\author{
Massimiliano Casaletti, Member, IEEE, Maciej Śmierzchalski, Mauro Ettorre, Senior Member, IEEE, \\ Ronan Sauleau, Senior Member, IEEE, and Nicolas Capet
}

\begin{abstract}
This paper deals with the design of scalar metasurface antennas capable of radiating a well-polarized beam in the far-field or near-field zones. The equivalent electric current over the metasurface is used to derive design equations to generate the desired field pattern based on the scalar impedance condition. In particular, it is shown that scalar metasurfaces can be used to generate linear and circular polarizations for a fixed pointing direction in the far field by properly changing locally the scalar impedance boundary condition. In addition, they can also be used to generate normal polarized Bessel beams in the nearfield region. Several solutions are presented at $20 \mathrm{GHz}$, with different polarizations and feeders developed in the framework of a two-year research project financed by the French space agency (Centre National d'Etudes Spatiales). Measurements and full-wave results validate the proposed approach.
\end{abstract}

Index Terms-Leaky waves (LWs), metasurface antenna, periodic surface, surface waves (SWs).

\section{INTRODUCTION}

M ETASURFACES are the 2-D equivalents of metamaterials [1]. They consist of textured scatterers/apertures, arranged along a surface that can be, in general, the surface of a nonuniform stratified medium. The properties of these surfaces are described in terms of tensorial or scalar surface impedances (analogous to the constitutive parameters for volumetric metamaterials). Metasurfaces have been recently used in many applications like holographic antennas [2], leaky-wave (LW) antennas [3]-[5] planar lenses [6]-[8], polarization convertors [9], [10], orbital angular momentum communication [11], transformation optics [12], [13] or future mathematical operation [14].

All these works are based on the pioneering results of Oliner and Hessel [15] about surface wave (SW) propagation over a sinusoidally modulated impedance. By choosing an appropriate modulated surface impedance, it is possible to control the propagation of SW along a surface or to obtain the transition from $\mathrm{SW}$ to $\mathrm{LW}$ modes in order to realize antennas [1], [16].

M. Casaletti is with the Laboratoire d'Electronique et d'Electromagnétisme, Unité de Recherche 2, Sorbonne Universités, Pierre-and-Marie-Curie University, Paris 75005, France (e-mail: massimiliano.casaletti@upmc.fr).

M. Smierzchalski, M. Ettorre, and R. Sauleau are with the Institute of Electronics and Telecommunications of Rennes, University of Rennes 1, Rennes 35042, France (e-mail: maciej.smierzchalski@univ-rennes1.fr; mauro.ettorre@univ-rennes1.fr; ronan.sauleau@univ-rennes1.fr).

N. Capet is with the Centre National d'Études Spatiales, Antenna Department, Toulouse 31400, France (e-mail: nicolas.capet@cnes.fr).
Surfaces composed by symmetric subwavelength printed elements over grounded dielectric slabs have been largely used due to the possibility to obtain modulated scalar impedances by locally changing the element dimension [1]-[14]. Some works like [1] and [17] make use of anisotropic elements to obtain tensorial impedances in order to control the polarization of the radiated field. The latter solution leads to more sophisticated design procedures [18].

Our objective is to propose a simple procedure for the design of metasurface antennas radiating polarized beam pointing to a general tilted direction in the far-field or broadside in the near-field region [20], [21]. The choice of using a scalar metasurface simplifies the impedance design process (synthesis of an impedance parameter instead of three as for the tensorial case) that can be crucial for future reconfiguration processes. The antenna design is based on the equivalent magnetic current radiation integral. This approach can be considered as an extension of [3] to an arbitrary radiation pattern. The advantage with respect to the scalar solution in [2] is the possibility to take into account polarization effects associated with the source.

This paper, summarizing the two years' results of this project activity, is structured as follows. Section II summarizes the basic phenomenology and the design guidelines. Section III presents the details of the aperture amplitude synthesis for far-field applications. Section IV provides the main results obtained for these antennas relevant to far-field applications. Section V presents an alternative design procedure for the optimization of the antenna aperture efficiency. The formulation and results obtained for near-field applications are described in Section VI. Conclusions are drawn in Section VII.

\section{Problem Formulation}

\section{A. Background}

In antenna applications, the modulated surface impedance is used to transform an SW, launched by a feeder, into an LW mode by using a locally periodic modulation.

In the following, we suppose that the feeder launches an incident SW propagating in a general direction on a metasurface placed in the $x y$ plane (Fig. 1). For a lossless nonmodulated inductive scalar surface impedance $Z_{s}=j \bar{X}_{s}$ $\left(\bar{X}_{s}>0\right)$, the dominant SW mode has a $\mathrm{TM}_{z}$ polarization (where $z$ is the normal to the surface and TM stands for transverse magnetic).

The magnetic field on the surface is assumed to be of the general form

$$
\mathbf{H}_{\text {aperture }}^{\text {inc }}\left(\boldsymbol{\rho}^{\prime}\right)=A^{\mathrm{TM}}\left(\boldsymbol{\rho}^{\prime}\right) e^{-j \hat{\mathbf{k}}_{\mathbf{t}}\left(\rho^{\prime}\right) \cdot \rho^{\prime}} \hat{\mathbf{h}}\left(\boldsymbol{\rho}^{\prime}\right)
$$




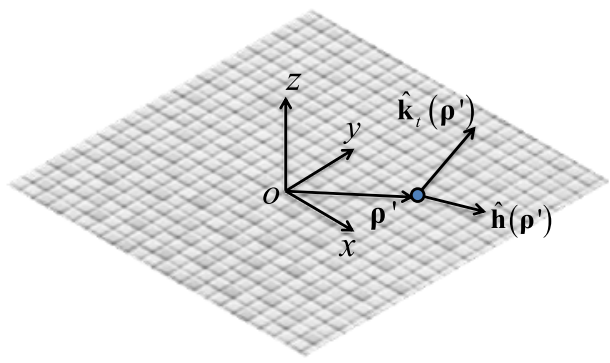

Fig. 1. Geometry of the problem.

where $\boldsymbol{\rho}^{\prime}=x^{\prime} \hat{\mathbf{x}}+y^{\prime} \hat{\mathbf{y}}$ is a general point on the antenna surface, $A^{\mathrm{TM}}$ the amplitude, $\hat{\mathbf{h}}$ the polarization unit-vector, $\overline{\mathbf{k}}_{\mathbf{t}}=\bar{k}_{t} \hat{\mathbf{k}}_{t}$, and $\hat{\mathbf{k}}_{\mathbf{t}}$ the propagation direction.

The propagation constant $\bar{k}_{t}$ is obtained by solving the transverse dispersion problem [19] as

$$
\bar{k}_{t}=k_{0} \sqrt{1+\left(\bar{X}_{s}\left(\overline{\mathbf{k}}_{\mathbf{t}}\right) / \zeta\right)^{2}}>k_{0}
$$

where $\zeta$ and $k_{0}=\omega \sqrt{\varepsilon_{0} \mu_{0}}$ are the free space impedance and wave-number, respectively, and it has been pointed out that, in general, the impedance depends on wavenumber (spatial dispersion).

The corresponding tangent electric field is obtained by using the scalar impedance boundary condition

$$
\begin{aligned}
\mathbf{E}_{\text {aperture }}^{\text {inc }}\left(\rho^{\prime}\right) & =-Z_{S}\left(\boldsymbol{\rho}^{\prime}\right) \hat{\mathbf{z}} \times \mathbf{H}_{\text {aperture }}^{\text {inc }}\left(\rho^{\prime}\right) \\
& =-A^{\mathrm{TM}}\left(\boldsymbol{\rho}^{\prime}\right) Z_{S}\left(\boldsymbol{\rho}^{\prime}\right) \hat{\mathbf{z}} \times \hat{\mathbf{h}}\left(\boldsymbol{\rho}^{\prime}\right) e^{-j \overline{\mathbf{k}}_{\mathbf{t}}\left(\boldsymbol{\rho}^{\prime}\right) \cdot \rho^{\prime}} .
\end{aligned}
$$

Let us consider now a local small periodic modulation of the surface impedance in the propagation direction (small perturbation of the boundary condition) of the form

$$
Z_{s}\left(\boldsymbol{\rho}^{\prime}\right)=j \bar{X}_{s}\left(1+M\left(\boldsymbol{\rho}^{\prime}\right) \cos \left(\frac{2 \pi}{p\left(\boldsymbol{\rho}^{\prime}\right)} \hat{\mathbf{k}}_{t}\left(\boldsymbol{\rho}^{\prime}\right) \cdot \boldsymbol{\rho}^{\prime}\right)\right)
$$

where $M$ and $p$ are the local modulation index and periodicity, respectively. Under the assumption that the impedance variation is sufficiently slow, the propagation problem can now be addressed using a periodic quasi-geometrical optics approximation (or equivalently a local tangent periodic modulation problem [1], [3]) as shown in the appendix. It turns out that (1) and (3) can be still considered as a good approximation of the field [15], while (2) has to be replaced by the solution of the following continued fraction dispersion equation [15]:

$$
\begin{gathered}
1-\frac{j \zeta_{0}}{X_{S}} \sqrt{1-\left[\frac{k_{t}}{k_{0}}+\frac{2 \pi n}{k_{0} p}\right]^{2}} \\
=\frac{M^{2} / 4}{1-\frac{j \zeta_{0}}{X_{S}} \sqrt{1-\left[\frac{k_{t}}{k_{0}}+\frac{2 \pi(n-1)}{k_{0} p}\right]^{2}}} \\
-\frac{M^{2} / 4}{1-\frac{j \zeta_{0}}{X_{S}} \sqrt{1-\left[\frac{k_{t}}{k_{0}}+\frac{2 \pi(n-2)}{k_{0} p}\right]^{2}}}-\cdots \\
+\frac{M^{2} / 4}{1-\frac{j \zeta_{0}}{X_{S}} \sqrt{1-\left[\frac{k_{t}}{k_{0}}+\frac{2 \pi(n+1)}{k_{0} p}\right]^{2}}}
\end{gathered}
$$

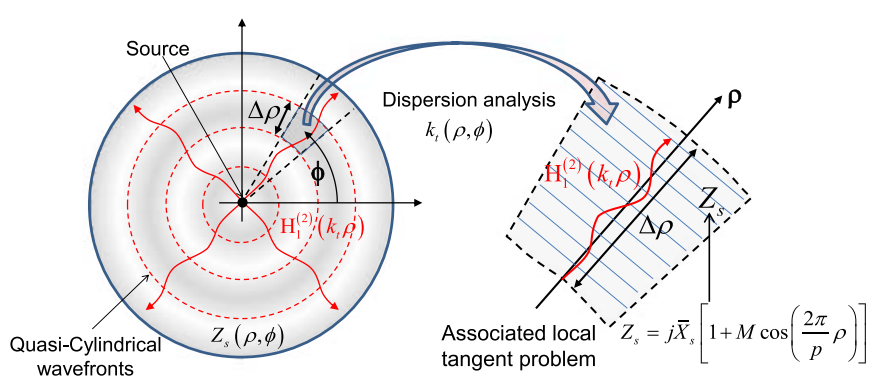

(a)

(b)

Fig. 2. Improved incident magnetic field approach geometry. (a) Antenna geometry. (b) Local tangent problem.

$$
-\frac{M^{2} / 4}{1-\frac{j \zeta_{0}}{X_{S}} \sqrt{1-\left[\frac{k_{t}}{k_{0}}+\frac{2 \pi(n+2)}{k_{0} p}\right]^{2}}}-\cdots
$$

The propagation constant becomes a complex quantity; thus, part of the energy is radiated in space (LW mode). However, as stated in [1] and [15], the modulated propagation constant can be approximated as

$$
k_{t}\left(\rho^{\prime}\right) \simeq \bar{k}_{t}\left(\rho^{\prime}\right)
$$

for the estimation of the incident magnetic field.

\section{B. Improved Incident Magnetic Field Formulation}

The variation of $M$ and $p$ over the surface can introduce a phase and amplitude deviation from (6). Thus, to increase the accuracy of the estimation of the incident magnetic field (1), we introduce here a new approach. The idea, based on the periodic Geometrical Optics (GO) approximation, is to decompose the metasurface into various subdomains in the propagation direction, in which the surface modulation (4) is assumed constant ( $M$ and $p$ are constant). As an example, we consider a metasurface excited by the cylindrical wave, from (A4) and (A7) with a small impedance modulation $\hat{\mathbf{k}}_{t}\left(\rho^{\prime}\right)=\left(\nabla_{t} S\left(\rho^{\prime}\right) /\left\|\nabla_{t} S\left(\rho^{\prime}\right)\right\|\right) \simeq \hat{\rho}, \forall \rho^{\prime}$. The corresponding geometry is reported in Fig. 2(a).

For each direction $\phi$, several associated local tangent problems with radial direction of propagation can be defined as shown in Fig. 2(a) and (b).

In each small sectorial area [shown in Fig. 2(b)] where the above-mentioned modulation quantities can be considered constant, the complex propagation constant $k_{t}$ can be found using (5). Thus, assuming to decompose the radial dimension in $N$ sectors (of dimension $\Delta \rho$ ), the incident magnetic field in the $n$th sector can be approximated as

$$
\mathbf{H}_{\text {inc }}=A_{n, \phi}^{\mathrm{TM}} H_{1}^{(2)}\left(k_{t}^{n, \phi} \rho\right) \hat{\boldsymbol{\phi}}
$$

where $k_{t}^{n, \phi}$ is obtained by solving the exact dispersion relation (5) of the local tangent problem associated with $(n-1)$ th sector. In the process, we assume for the first sector $k_{t}^{1, \phi}=\bar{k}_{t}$, while the complex amplitude $A_{n, \phi}^{\mathrm{TM}}$ is obtained by imposing the continuity of the field across two adjacent sectors

$$
A_{n, \phi}^{\mathrm{TM}}=\frac{A_{n-1, \phi}^{\mathrm{TM}} H_{1}^{(2)}\left(k_{t}^{n-1, \phi} n \Delta \rho^{\prime}\right)}{H_{1}^{(2)}\left(k_{t}^{n, \phi} n \Delta \rho^{\prime}\right)} .
$$




\section{Radiated Field}

The field radiated by the locally modulated metasurface can be expressed as the field radiated by the equivalent surface magnetic currents, defined as

$$
\mathbf{J}_{M}\left(\mathbf{r}^{\prime}\right)=2 \mathbf{E}\left(\mathbf{r}^{\prime}\right) \times \hat{\mathbf{z}}=-2 A^{\mathrm{TM}}\left(\boldsymbol{\rho}^{\prime}\right) Z_{s}\left(\boldsymbol{\rho}^{\prime}\right) e^{-j \mathbf{k}_{\mathbf{t}}\left(\boldsymbol{\rho}^{\prime}\right) \cdot \boldsymbol{\rho}^{\prime}} \hat{\mathbf{h}}
$$

where the amplitude term $A^{\mathrm{TM}}\left(\rho^{\prime}\right)$ and the propagation constant $k_{\mathbf{t}}\left(\rho^{\prime}\right)$ are those obtained using the procedure in Section II-B, while the direction is dictated by the source. Using (4) in (9), we have

$$
\begin{aligned}
\mathbf{J}_{M}\left(\mathbf{r}^{\prime}\right)=-A^{\mathrm{TM}}\left(\boldsymbol{\rho}^{\prime}\right) \hat{\mathbf{h}}\{ & 2 \bar{X}_{s} e^{-j k_{\mathbf{t}} \hat{\mathbf{k}}_{t}\left(\boldsymbol{\rho}^{\prime}\right) \cdot \boldsymbol{\rho}^{\prime}} \\
& +\bar{X}_{S} M\left(\boldsymbol{\rho}^{\prime}\right) e^{-j\left(k_{\mathbf{t}}\left(\boldsymbol{\rho}^{\prime}\right)-\frac{2 \pi}{p\left(\rho^{\prime}\right)}\right) \hat{\mathbf{k}}_{t}\left(\boldsymbol{\rho}^{\prime}\right) \cdot \boldsymbol{\rho}^{\prime}} \\
& \left.+\bar{X}_{S} M\left(\boldsymbol{\rho}^{\prime}\right) e^{-j\left(k_{\mathbf{t}}\left(\boldsymbol{\rho}^{\prime}\right)+\frac{2 \pi}{p\left(\rho^{\prime}\right)}\right) \hat{\mathbf{k}}_{t}\left(\boldsymbol{\rho}^{\prime}\right) \cdot \boldsymbol{\rho}^{\prime}}\right\} \\
= & \mathbf{J}_{M}^{(0)}\left(\mathbf{r}^{\prime}\right)+\mathbf{J}_{M}^{(-1)}\left(\mathbf{r}^{\prime}\right)+\mathbf{J}_{M}^{(1)}\left(\mathbf{r}^{\prime}\right) .
\end{aligned}
$$

The first and third terms in (10) represent nonradiative currents (associated with SW), while the second one can be radiative if $k_{\mathbf{t}}\left(\boldsymbol{\rho}^{\prime}\right)-\left(2 \pi / p\left(\boldsymbol{\rho}^{\prime}\right)\right)<k_{0}$.

By changing locally the period of the modulated scalar impedance, it becomes possible to transform the SW into an LW pointing to a desired direction.

The radiated far field can be obtained using the term $\mathbf{J}_{M}^{(-1)}\left(\mathbf{r}^{\prime}\right)$ of (10) in the radiating integral

$$
\mathbf{E}^{\mathrm{FF}}(\theta, \phi)=j k_{0} \iint_{S} \hat{\mathbf{r}}(\theta, \phi) \times \mathbf{J}_{M}^{(-1)}\left(\mathbf{r}^{\prime}\right) e^{j k_{0} \mathbf{r}^{\prime} \cdot \hat{\mathbf{r}}} d \mathbf{r}^{\prime}
$$

where $S$ is the antenna's surface.

For cylindrical wave excitations, (11) reduces to

$$
\begin{aligned}
\mathbf{E}^{\mathrm{FF}}(\theta, \phi)= & -j k_{0} \iint_{S}\left[-\hat{\theta} \cos \left(\phi-\phi^{\prime}\right)+\hat{\boldsymbol{\phi}} \sin \left(\phi-\phi^{\prime}\right)\right] \\
& \times J_{M \phi}\left(\rho^{\prime}, \phi^{\prime}\right) e^{j k_{0} \rho^{\prime} \sin \theta \cos \left(\phi-\phi^{\prime}\right)} d \phi^{\prime} d \rho^{\prime} .
\end{aligned}
$$

Equations (11) and (12) represent the basic block of our procedure. A desired radiation pattern could be obtained by deriving the appropriate magnetic current distribution in (11) and (12). Once the magnetic current distribution is found (analytically or numerically), the impedance is obtained by a two-step procedure.

1) The metasurface period $p\left(\rho^{\prime}\right)$ is obtained by imposing a phase-matching between the optimal current phase and $\mathbf{J}_{M}^{(-1)}\left(\mathbf{r}^{\prime}\right)$.

2) The average impedance $\bar{X}_{s}$ and the modulation index $M$ are found to match as much as possible the optimal amplitude law.

\section{FAR-Field Polarized BEAms ANTENNA}

In the following, we assume that the feeder can be approximated by a point source placed in the origin; thus, the incident SW has a cylindrical wavefront:

$$
\mathbf{H}_{\text {aperture }}^{\mathrm{inc}}=A_{\mathrm{TM}} H_{1}^{(2)}\left(k_{t} \rho\right) \hat{\boldsymbol{\phi}}
$$

and the corresponding equivalent magnetic current will be directed in the $\hat{\boldsymbol{\phi}}$-direction

$$
\mathbf{J}_{M}\left(\mathbf{r}^{\prime}\right)=J_{M \phi} \hat{\boldsymbol{\phi}}=A_{\mathrm{TM}} Z_{S}\left(\mathbf{r}^{\prime}\right) H_{1}^{(2)}\left(k_{t}\left(\mathbf{r}^{\prime}\right) \rho\right) \hat{\boldsymbol{\phi}} .
$$

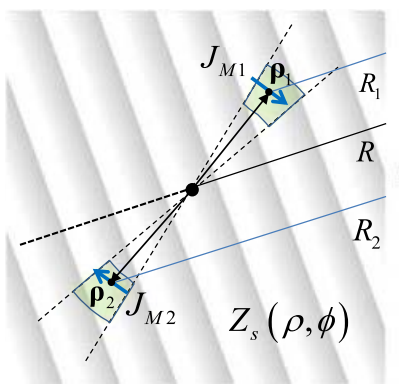

(a) (b)

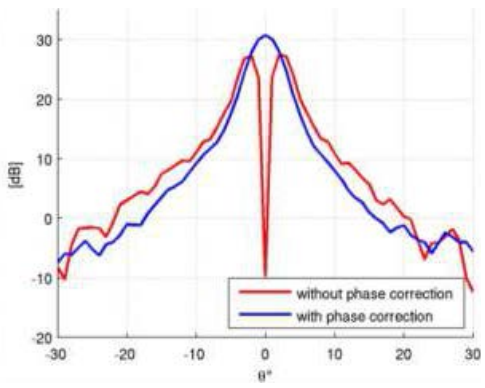

Fig. 3. (a) Surface current distribution over the metasurface without phase correction. (b) Comparison of the radiated far-field for a broadside antenna with (blue line) and without (red line) $90^{\circ}$ phase correction.

\section{A. Linearly Polarized Antenna}

A linearly polarized (LP) metasurface antenna pointing at $\theta_{0}, \phi_{0}$ can be obtained by realizing a linear phasing of the equivalent surface currents. However, due to $\hat{\phi}$-polarization of the equivalent magnetic current, symmetric modulations with respect to the incident field phase center have to be avoided since leading to a null of the radiated far field in the desired maximum direction [Fig. 3(a) and (b)].

On the other hand, introducing a $90^{\circ}$ phase shift between two symmetric surface current elements leads to a coherent sum of each contribution in the far field, thus obtaining a tilted beam in the desired direction $\theta_{0}, \phi_{0}$

$$
J_{M}\left(\rho^{\prime}, \phi^{\prime}\right)= \begin{cases}A\left(\rho^{\prime}\right) \hat{\boldsymbol{\phi}}, & \text { if } 0 \leq \phi_{0}-\phi^{\prime}<\pi \\ -A\left(\rho^{\prime}\right) \hat{\boldsymbol{\phi}}, & \text { if } \pi \leq \phi_{0}-\phi^{\prime}<2 \pi .\end{cases}
$$

In fact, considering the magnetic current distribution (15) on a circular aperture of radius $R_{a}$ exited by a point source $\left(A\left(\rho^{\prime}\right)=\left(2 / \pi k_{t} \rho^{\prime}\right)^{1 / 2}\right)$ in (14) and calculating the radiated field in the $\phi=\phi_{0}$ cut-plane gives

$$
\mathbf{E}^{\mathrm{FF}}\left(\theta, \phi_{0}\right)=-\hat{\boldsymbol{\phi}} 16 j k_{0} \sqrt{\frac{2 R_{a}}{\pi k_{t}}} \operatorname{sinc}\left(\frac{k_{0}}{\pi} R_{a}\left(\sin \theta-\sin \theta_{0}\right)\right) .
$$

The latter configuration can be obtained by using a modulated metasurface of the form (4) where the periodicity is a function of the azimuth angle

$$
\begin{aligned}
& p\left(\boldsymbol{\rho}^{\prime}\right) \\
& \quad= \begin{cases}\frac{2 \pi}{\left|k_{0} \sin \theta_{0} \cos \left(\phi_{0}-\phi\right)-k_{t}\right|}, & \text { if } 0 \leq \phi_{0}-\phi^{\prime}<\pi \\
-\frac{2 \pi}{\left|k_{0} \sin \theta_{0} \cos \left(\phi_{0}-\phi\right)-k_{t}\right|}, & \text { if } \pi \leq \phi_{0}-\phi^{\prime}<2 \pi .\end{cases}
\end{aligned}
$$

Fig. 4(a) and (b) shows the reactance chart metasurfaces obtained for an LP antenna pointing at $\theta_{0}=45^{\circ}, \phi_{0}=0^{\circ}$ and at $\theta_{0}=45^{\circ}, \phi_{0}=30^{\circ}$, respectively.

\section{B. Circularly Polarized Antenna}

A scalar circularly polarized (CP) metasurface antenna pointing at $\theta_{0}, \phi_{0}$ can be obtained by phasing the azimuthal magnetic surface current as

$$
J_{M}\left(\rho^{\prime}, \phi^{\prime}\right)=e^{ \pm j \phi^{\prime}} e^{-j k_{0} \rho^{\prime} \sin \theta_{0} \cos \left(\phi^{\prime}-\phi_{0}\right)} \hat{\boldsymbol{\phi}}
$$




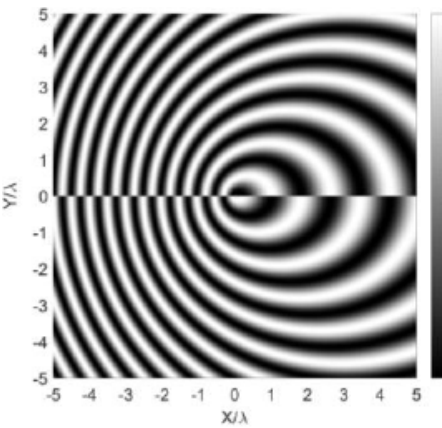

(a)

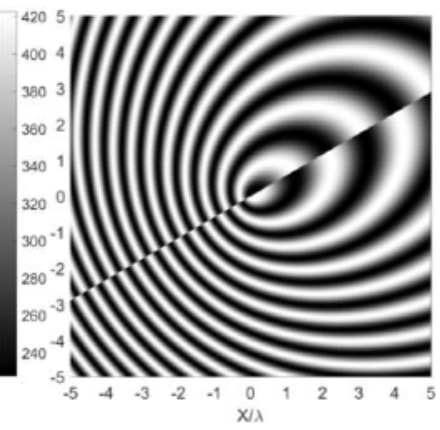

(b)
Fig. 4. Reactance chart for a LP beam pointing at (a) $\theta_{0}=45^{\circ}, \phi_{0}=0^{\circ}$ and (b) $\theta_{0}=45^{\circ}, \phi_{0}=30^{\circ}$. The average impedance is $Z_{s}=j 290 \Omega$ and modulation index $M=0.17$.

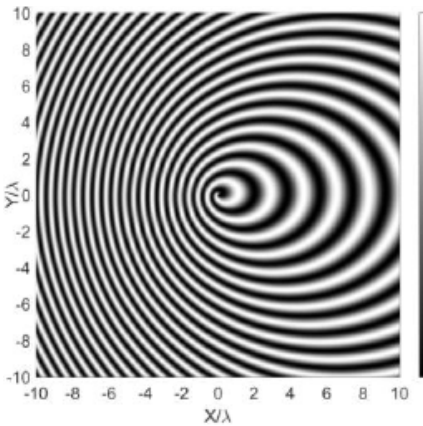

(a)

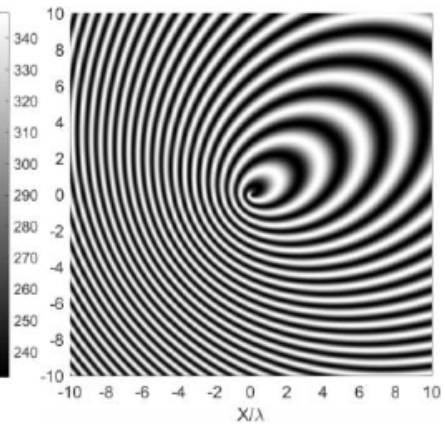

(b)
Fig. 5. Reactance chart for a LH-CP beam pointing at (a) $\theta_{0}=45^{\circ}, \phi_{0}=0^{\circ}$ and (b) $\theta_{0}=60^{\circ}, \phi_{0}=30^{\circ}$. The average impedance is $Z_{s}=j 290 \Omega$ and modulation index $M=0.17$.

where the upper and lower indexes give rise to left-hand and right-hand circular polarizations, respectively. The linear phasing in (18) allows having coherent optical paths for each surface current element at the desired pointing direction, while the term $e^{ \pm j \phi^{\prime}}$ handles the polarization. In fact, considering a circular aperture of radius $R_{a}$, using (18) in the radiation integral (12), and observing at $\theta_{0}, \phi_{0}$ leads to a CP field

$$
\mathbf{E}^{\mathrm{FF}}\left(\theta_{0}, \phi_{0}\right)=j \frac{k R_{a} \pi e^{-j \phi_{p}}}{2}[\hat{\theta} \pm j \hat{\boldsymbol{\phi}}] .
$$

The corresponding impedance law can be obtained substituting in (4) the following relation:

$$
\begin{aligned}
\cos & \left(\frac{2 \pi}{p\left(\boldsymbol{\rho}^{\prime}\right)} \rho^{\prime}\right) \\
& =\operatorname{Re}\left\{e^{j \operatorname{Arg}\left(H_{1}^{(2)}\left(k_{t} \rho\right)\right)} e^{\mp j \phi^{\prime}} e^{j k_{0} \rho^{\prime} \sin \theta_{0} \cos \left(\phi^{\prime}-\phi_{0}\right)}\right\} \\
& \simeq \cos \left(k_{t} \rho \mp j \phi^{\prime}+k_{0} \rho^{\prime} \sin \theta_{0} \cos \left(\phi^{\prime}-\phi_{0}\right)\right) .
\end{aligned}
$$

As an example, Fig. 5(a) and (b) represents the reactance chart for a $\mathrm{CP}$ metasurface antenna point at $\theta_{0}=45^{\circ}, \phi_{0}=0^{\circ}$ and at $\theta_{0}=45^{\circ}, \phi_{0}=30^{\circ}$, respectively, and operating at $20 \mathrm{GHz}$.

\section{EXPERIMENTAL DEMONSTRATIONS FOR FAR-FIELD APPLICATIONS}

Two different solutions operating at $20 \mathrm{GHz}$ are presented here, a CP and an LP antenna, both pointing at $\theta_{0}=45^{\circ}$,

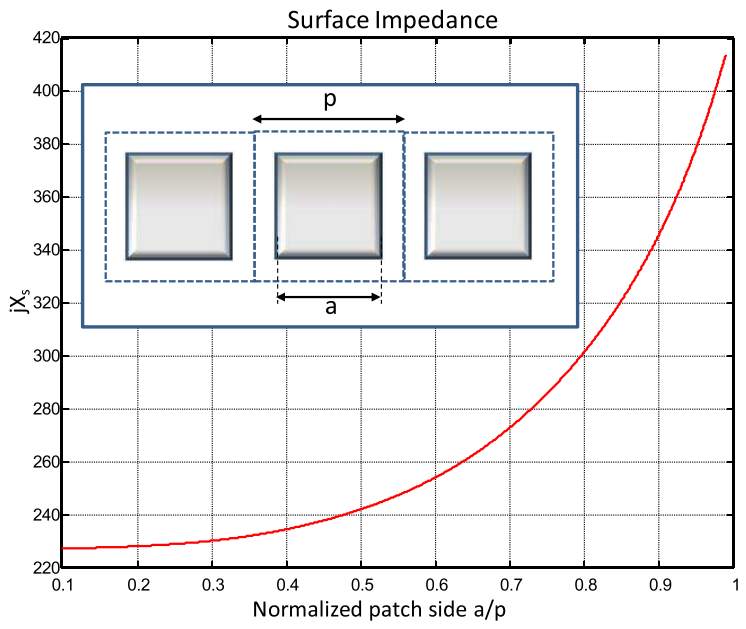

Fig. 6. Impedance as a function of normalized dimension for a printed squared patch over a grounded dielectric slab $\left(\varepsilon_{r}=3.66, h=1.52 \mathrm{~mm}\right)$.

$\phi_{0}=0^{\circ}$. The surface impedance is realized by printing squared patches on a squared lattice of side $1.25 \mathrm{~mm}$ on a grounded dielectric slab (Rogers $4350 \mathrm{~b}$ with a relative permittivity of 3.66 and a thickness of $1.52 \mathrm{~mm}$ ). The variation of the impedance as a function of the patch dimension for a $\mathrm{TM}_{z} \mathrm{SW}$ is reported in Fig. 6.

The cylindrical SW is launched by a pin with a metallic cap on the top similar to that proposed in [3].

For the correct design of a metasurface antenna, it is crucial to understand if all the energy associated with the SW is transformed into a LW, for two main reasons:

1) The energy remained confined into SW can be considered lost for the radiated field (lower gain).

2) The SW that reaches the edge of the antenna will be reflected back toward the source (radiating in a different direction) and diffracted in space, thus spoiling the radiation pattern.

We used the full-wave software Ansys HFSS to study the behavior of the Poynting vector traveling inside the substrate. This enables us to find the best compromise between minimum modulation index and attenuation of the SW at the antenna edges. An example of amplitude of the radial component of the pointing vector relative to the $\mathrm{CP}$ antenna is represented in Fig. 7 for four values of $M$ (from 0.14 to 0.2 ). The SW propagating in the positive $x$-direction (forward radiation) has a lower attenuation constant compared to the one propagating in the opposite direction (backward radiation). To ensure satisfactory aperture efficiency, a proper transition between SW and LW must be selected, and a low modulation index is preferred. On the other hand, a high index is desirable to minimize contributions of SW at the antenna edges.

All the solutions presented in this paper have been designed considering the minimum size of the fabricated square metallic patch equal to $500 \mu \mathrm{m}$ as required by fabrication constraints.

\section{A. CP Antenna Pointing at $\theta_{0}=45^{\circ}, \phi_{0}=0^{\circ}$}

Since the attenuation constant of the LW depends on its nature (forward or backward radiation), an elliptical panel with a semimajor axis of $10 \lambda$ and a semiminor axis of $7.5 \lambda$ has 


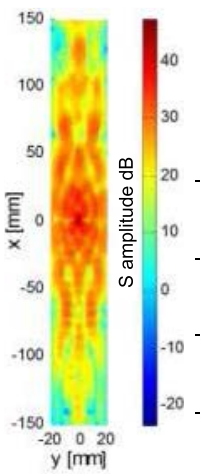

(a)

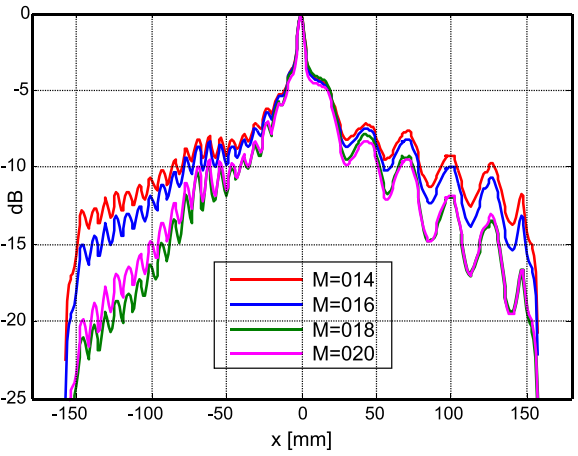

(b)
Fig. 7. (a) 2-D plot of the Poynting vector amplitude inside the dielectric substrate for a $10 \lambda$ antenna with metasurface $\{f=20 \mathrm{GHz}, \lambda=15 \mathrm{~mm}$, $X_{S}=290 \Omega$, and $\left.M=0.18\right\}$. (b) $x$-axis cut-plane for different modulations of metasurface $(0.14-0.2)$.

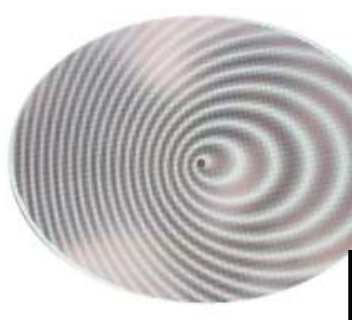

(a)

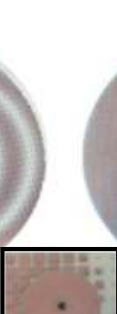

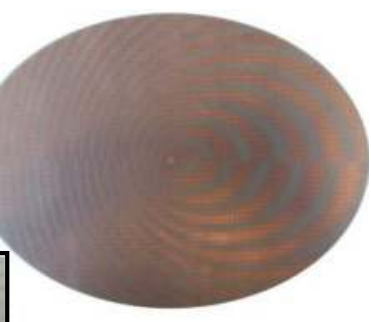

(b)
Fig. 8. (a) CP antenna final design. (b) LP antenna final design. Inset: feeder detail.

been selected in order to increase the effective aperture of the antenna. The metasurface has an average impedance of $Z_{s}=j 290 \Omega$ and the modulation index $M$ equals 0.18 . This ensures an attenuation of the incident SW of about $20 \mathrm{~dB}$ at the antenna edges. The antenna has been simulated and optimized using HFSS. The final geometry is shown in Fig. 8(a). Its simulated and measured reflection coefficients are represented in Fig. 9(a).

Fig. 9(b) represents the simulated and measured radiation patterns at the central frequency, while Fig. 9(c) shows the maximum gain in the frequency range between 19 and $21 \mathrm{GHz}$. The antenna gain has been measured by the comparison method using a $20-\mathrm{dBi}$ standard horn. It can be noticed that measurements and simulations are in good agreement. The maximum gain reaches $25.5 \mathrm{~dB}$ (24.4-dB simulation), and side lobe level $-15 \mathrm{~dB}$, with an aperture efficiency of $11 \%(6.8 \%)$. The lower values obtained in simulations may be due to the accuracy of the mesh discretization used for the numerical analysis.

\section{B. LP Antenna Pointing at $\theta_{0}=45^{\circ}, \phi_{0}=0^{\circ}$}

A panel similar to that for the $\mathrm{CP}$ antenna has been used to realize a LP antenna. The selected surface impedance modulation is plotted in Fig. 4(a) while its implementation is reported in Fig. 8(b). The measured and simulated results (reflection coefficient, radiation pattern at the central frequency, and maximum gain as a function of frequency) are shown in Fig. 10.

The maximum gain reached by the antenna is $22 \mathrm{~dB}$ (23.5-dB simulation) with a side lobe level lower than $15 \mathrm{~dB}$,

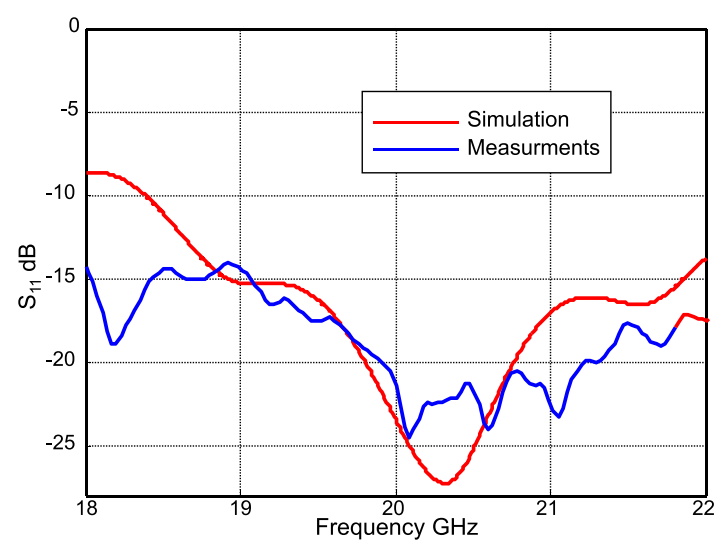

(a)

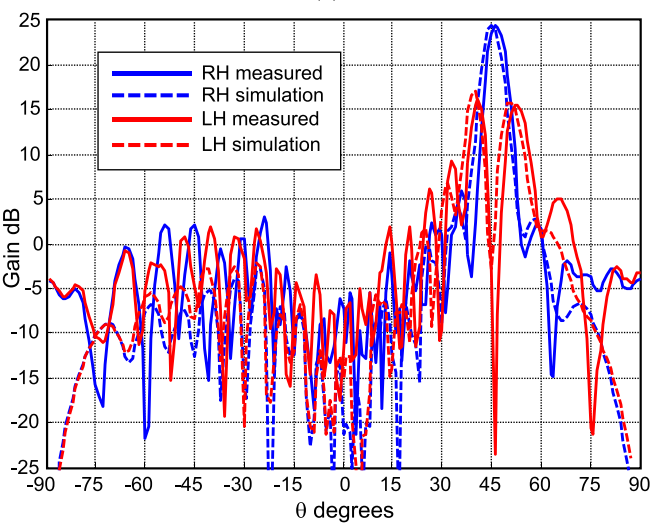

(b)

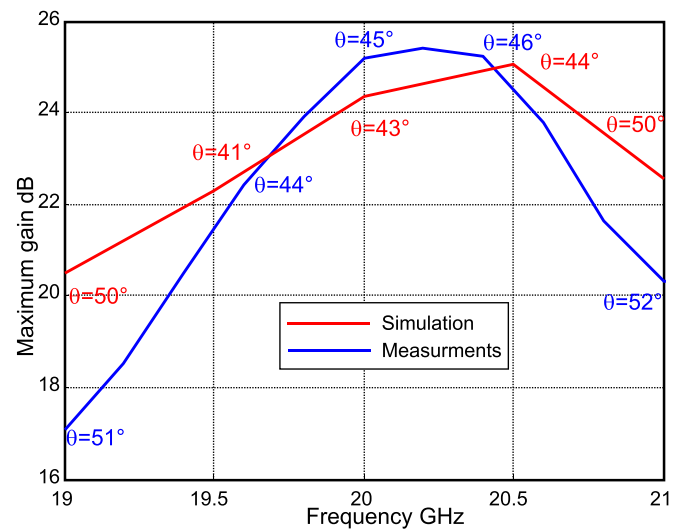

(c)

Fig. 9. (a) Measured and simulated reflection coefficients. (b) Measured and simulated copolarization Right-hand Circula Polarization and cross polarization Left-hand Circular Polarization components. (c) Measured and simulated gains in the frequency range spanning between 19 and $21 \mathrm{GHz}$. The main beam direction is also indicated.

which is in good agreement with the full wave simulations. The aperture efficiency is $3.5 \%$ (5\%).

\section{IMPROVEMENT OF APERTURE EFFICIENCY of Metasurface Antennas}

In this section, we present an alternative design for the LP tilted beam antenna. The aim is to improve the aperture efficiency introducing two innovations: a directive feeder and variable modulation index.

As can be seen in Figs. 4 and 5, the local period of the metasurface modulation depends on the azimuthal angle. This 


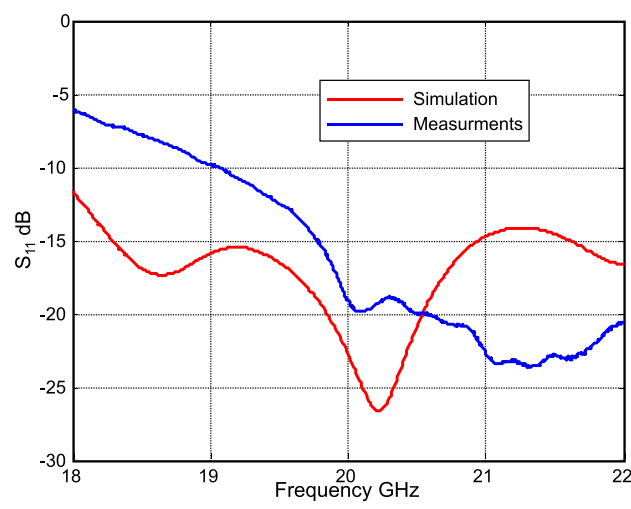

(a)

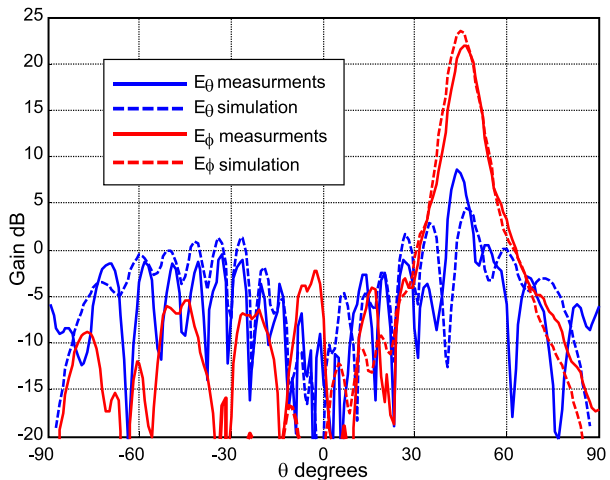

(b)

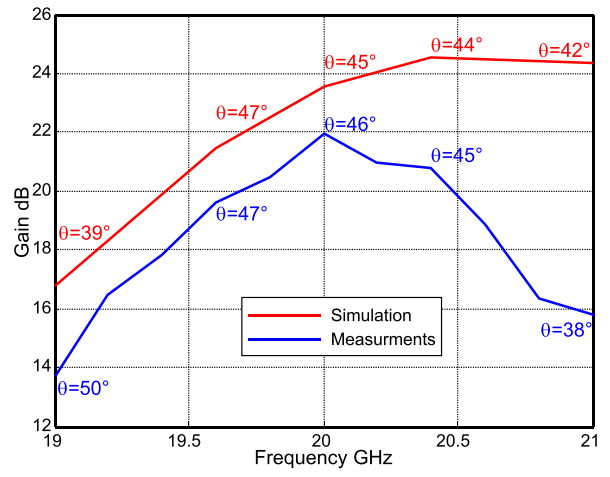

(c)

Fig. 10. (a) Measured and simulated reflection coefficients. (b) Measured and simulated copolarization $\left(E_{\theta}\right)$ and cross polarization $\left(E_{\varphi}\right)$ components. (c) Measured and simulated gains in the frequency range spanning between 19 and $21 \mathrm{GHz}$. The main beam direction is also indicated.

is due to the fact that the SW propagates from the feeder in radial direction; thus, some portions of antenna surface will radiate an LW in forward direction while others in backward direction (Floquet's mode \pm 1 ). Fig. 7 shows that the forward radiation needs a larger period $(1.5 \lambda)$ and has a smaller attenuation than the backward one $(0.75 \lambda)$.

The proposed solution makes use of a directional feeder in order to illuminate a specific portion of the metasurface defined by (17) having the desired radiating nature. The feeder [Fig. 11(a)] is inspired by the solutions presented in [22] and [23]. The tuning stubs have been deeply modified to make the design compact and metallic pin-made walls have been added to decrease the spurious back radiation. We opted to use only the backward radiation area of the metasurface because it allows an easy control of the imaginary parts

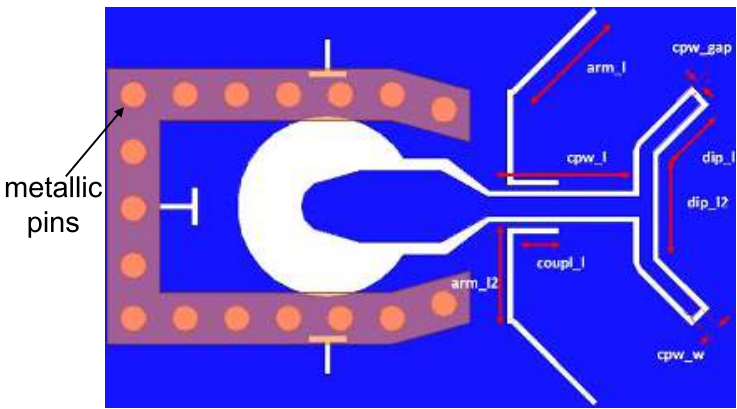

(a)

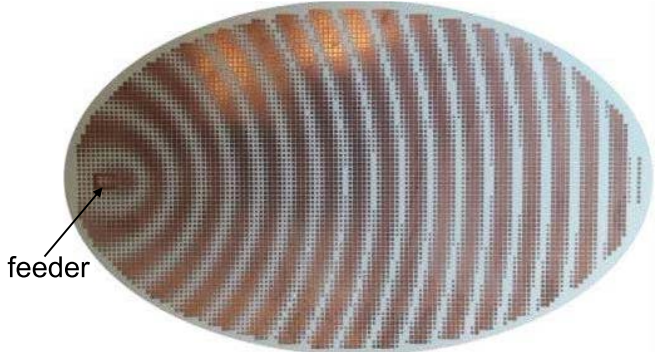

(b)

Fig. 11. (a) Directional feeder geometry: $c p w \_l=2.25 \mathrm{~mm}, c p w \_w=$ $0.3 \mathrm{~mm}$, arm_l$=1.9 \mathrm{~mm}$, arm_l$l 2=1.5 \mathrm{~mm}$, bend $=45^{\circ}$, dip_l $=1.1 \mathrm{~mm}$, dip_l$=0.87 \mathrm{~mm}$, and coupl_l$=0.8 \mathrm{~mm}$, via hole diameter $=0.4 \mathrm{~mm}$, pitch $=0.6 \mathrm{~mm}$. (b) Alternative LP antenna final design with nonuniform modulation index.

of the propagation constant acting on the modulation index [see Fig. 7(b)].

The final metasurface design [Fig. 11(b)] has been printed on an elliptical panel with a semimajor axis of $4 \lambda$ and a semiminor axis of $2.4 \lambda$. It has a modulation index $M$ varying between 0.15 and 0.3 with the radial distance from the feeder. This latter aspect gives rise to an efficient surface to LW transition (uniform leaked power) with acceptable attenuation of SW on the edges.

Fig. 12 represents the simulated and the measured results. The maximum gain is $20.3 \mathrm{~dB}(21.6-\mathrm{dB}$ simulation) corresponding to an aperture efficiency of $19 \%$ (24\%).

The LP antenna with directional feeder has a much narrower band than the one with an azimuthal invariant feeder. This is due to the use of only a backward radiating metasurface with a nonuniform modulation, increased dispersive behavior, and sensitivity to the metasurface modulation.

\section{NeAR-Field BeAm Antenna}

A near-field metasurface antenna can be realized by implementing a Bessel beam launcher. Bessel beams are nondiffractive exact solutions of Maxwell's equations [24]. In practice, these beams can be generated only in a limited area called nondiffractive range.

Let us consider a cylindrical coordinate system $(\rho, \phi, z)$. A Bessel beam polarized in the $z$-direction can be obtained by a magnetic current aperture distribution placed in $z=0$ of the form [25], [26]

$$
\mathbf{J}_{M}\left(\mathbf{r}^{\prime}\right)=A J_{1}\left(\beta_{\rho}^{B} \rho^{\prime}\right) \hat{\boldsymbol{\phi}}
$$

where $A$ is an amplitude constant, $J_{1}$ is the first-order Bessel function of the first kind, and $\beta_{\rho}^{B}<k_{0}$ is the transverse 


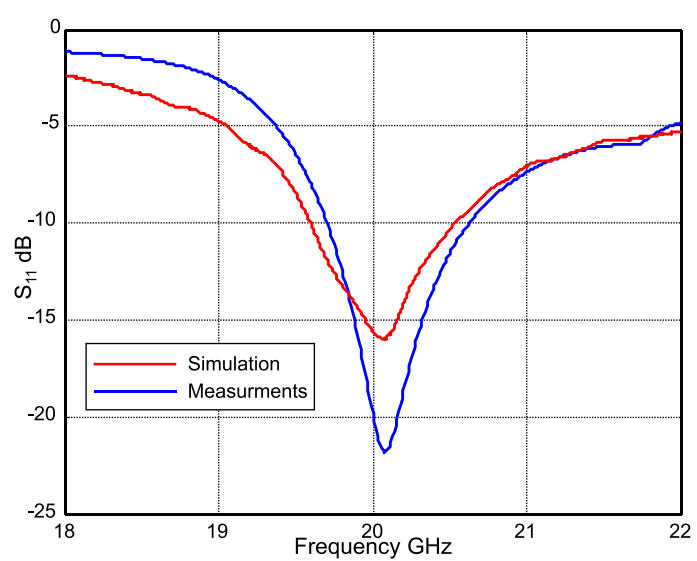

(a)

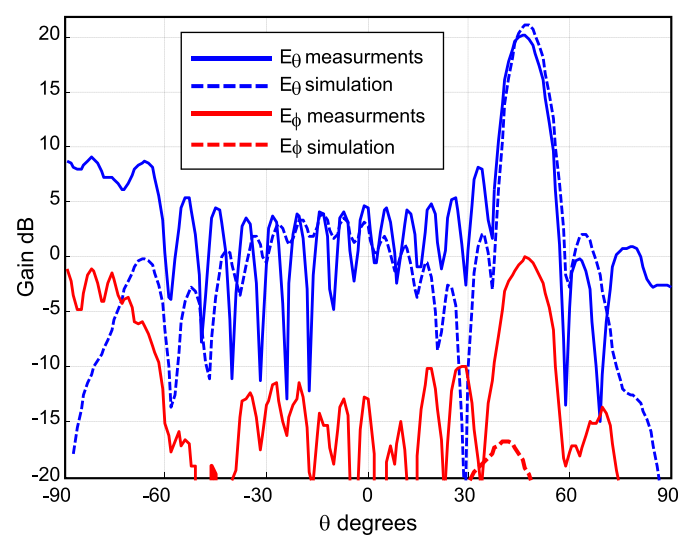

(b)

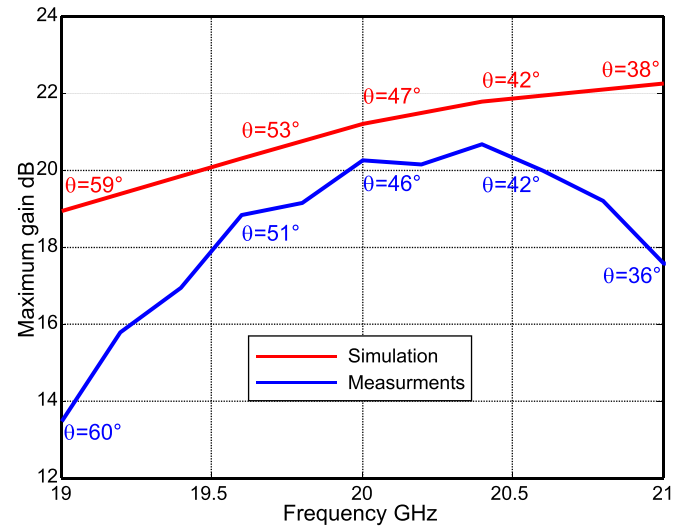

(c)

Fig. 12. (a) Measured and simulated reflection coefficients. (b) Measured and simulated copolarization $\left(E_{\theta}\right)$ and cross polarization $\left(E_{\varphi}\right)$ components. (c) Measured and simulated gains in the frequency range spanning between 19 and $21 \mathrm{GHz}$. The main beam direction is also indicated.

propagation constant of the beam. This latter and the radius of the metasurface $\rho_{\max }$ are directly related to the nondiffractive range of the Bessel beam

$$
z_{\max }=\rho_{\max } \sqrt{\left(k_{0} / \beta_{\rho}^{B}\right)^{2}-1} .
$$

A current distribution with the same phase as (21) can be obtained from (4) using the following term in the impedance

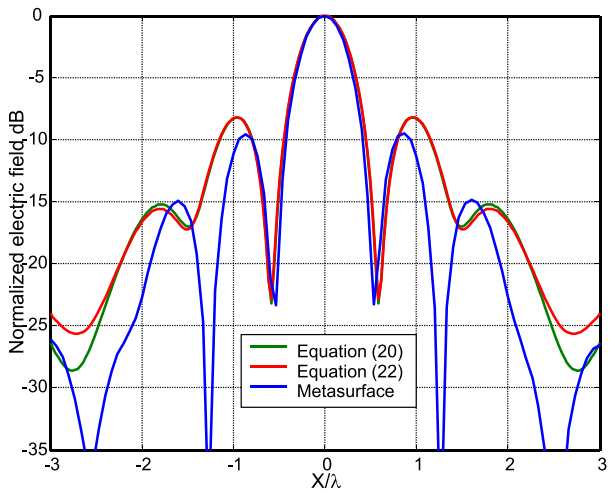

(a)

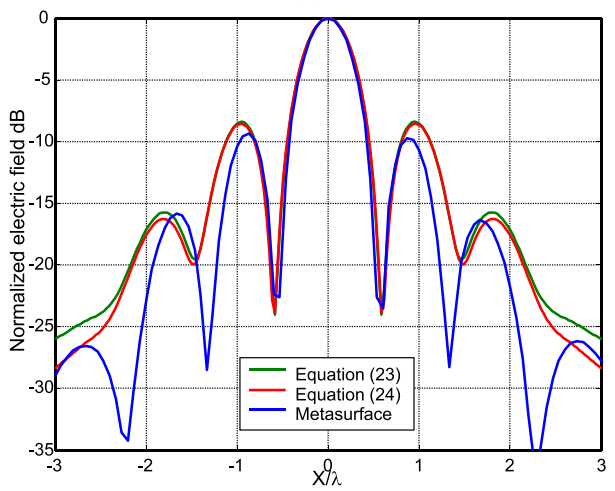

(b)

Fig. 13. (a) Near-field comparison at a distance of $2 \lambda$ from the antenna between an ideal Bessel beam, the field generated by an aperture described by (21), and its metasurface antenna implementation (23). (b) Same comparison using the aperture distribution (24) and its metasurface implementation (25).

expression:

$$
\begin{aligned}
& \cos \left(\frac{2 \pi}{p\left(\rho^{\prime}\right)} \rho^{\prime}\right) \\
& =\operatorname{Re}\left\{e^{j \operatorname{Arg}\left(H_{1}^{(2)}\left(k_{t} \rho^{\prime}\right)\right)} e^{-j \operatorname{Arg}\left(J_{1}\left(\beta_{\rho}^{B} \rho^{\prime}\right)\right)}\right\} \\
& \simeq \cos \left[-k_{t} \rho^{\prime}+\frac{3}{4} \pi-\pi \chi\left(-\cos \left(\beta_{\rho}^{B} \rho^{\prime}-\frac{3}{4} \pi\right)\right)\right]
\end{aligned}
$$

where

$$
\chi(x)= \begin{cases}1 & x \geq 0 \\ 0 & x<0\end{cases}
$$

is the step function.

It can be noted that (23) can also assure a similar amplitude law with respect to (21) since the Bessel and the Hankel functions have the same amplitude factor in their asymptotic expansion for large arguments. The two amplitude laws match exactly only in the case where $\beta_{\rho}^{B}=k_{t}$. The latter condition could not be satisfied since $\beta_{\rho}^{B}<k_{0}$ while $k_{t}>k_{0}$. The effect of this small amplitude error (tapering) is shown in Fig. 13(a) where a circular aperture of radius $3 \lambda$ illuminated with a Bessel function with $\beta_{\rho}^{B}=0.6 k_{0}$ has been considered. As can be seen, the above amplitude difference gives raise to small differences on the secondary lobes while the focusing effect is still present.

An alternative formulation has been proposed in [27] and [28], where the Bessel beam is obtained by 


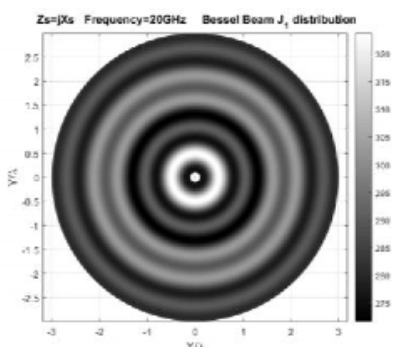

(a)

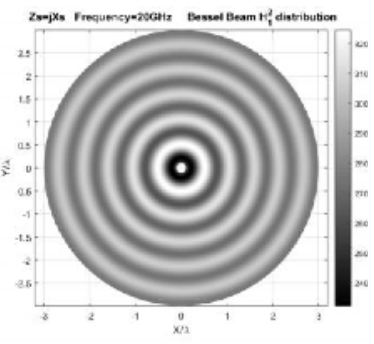

(b)
Fig. 14. Reactance chart for a Bessel beam polarized along $z$, generated with a $J_{1}$ distribution (a) or an $H_{1}^{(1)}$ distribution (b). The average impedance is $Z_{S}=j 290 \Omega$ and modulation index $M=0.17$.

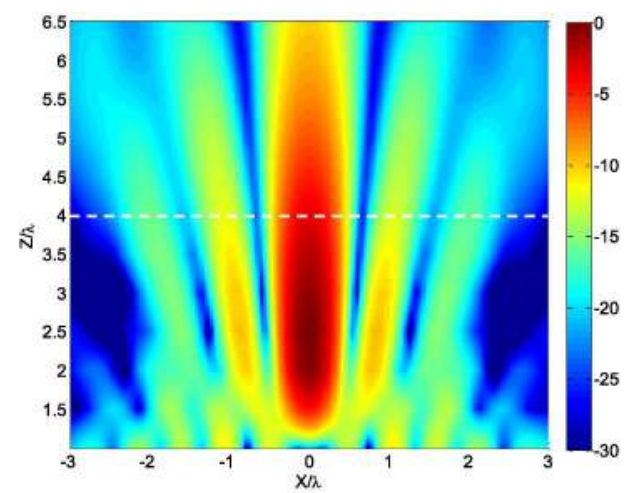

(a)

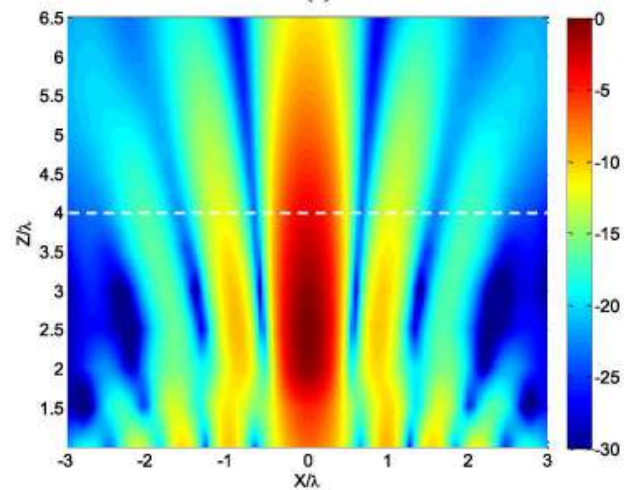

(b)

Fig. 15. Normalized near-field $E_{z}$ component radiated by the Bessel beam launcher antenna using (a) aperture distribution (21) and (b) alternative aperture distribution (24). The white lines indicate the limit of nondiffractive range.

an inward cylindrical wave distribution

$$
\mathbf{J}_{M}\left(\mathbf{r}^{\prime}\right)=A H_{1}^{(1)}\left(\beta_{\rho}^{B}\left(\mathbf{r}^{\prime}\right) \rho\right) \hat{\boldsymbol{\phi}}
$$

where $H_{1}^{(1)}$ is the first kind Hankel's function. Also for this case, a tapered distribution law (24) can be obtained (amplitude and phase) using the following expression:

$$
\begin{aligned}
\cos \left(\frac{2 \pi}{p\left(\rho^{\prime}\right)} \rho^{\prime}\right) & =\operatorname{Re}\left\{e^{j \operatorname{Arg}\left(H_{1}^{(2)}\left(k_{t} \rho^{\prime}\right)\right)} e^{-j \operatorname{Arg}\left(H_{1}^{(1)}\left(\beta_{\rho}^{B} \rho^{\prime}\right)\right)}\right\} \\
& \simeq \cos \left[\left(k_{t}-\beta_{\rho}^{B}\right) \rho^{\prime}\right] .
\end{aligned}
$$

Similar considerations on the aperture field amplitudes hold also for this configuration as shown in Fig. 13(b). Reactance chart examples for both formulations are shown in Fig. 14.

\section{Numerical Results}

The two near-field configurations presented in the previous section have been implemented using a circular panel of radius $3 \lambda$ sharing the same impedance parameters as the previous antennas. Since the panel dimension is smaller, a higher modulation index has been used close to the periphery of the antenna to prevent reflections.

The transverse propagation constant parameter of the Bessel beam has been selected as $\beta_{\rho}^{B}=0.6 k_{0}$; the corresponding nondiffracting zone defined by (22) is given by $z \leq 4 \lambda$.

Fig. 13 shows a comparison between the ideal Bessel beam, the tapered ideal beam, and the field radiated by the metasurface antenna at $2 \lambda$ from the antenna obtained with HFSS for both configurations. Good agreement has been obtained. Fig. 15 represents the map of the near field generated by the metasurface antenna. As can be seen, the focusing effect is present within the nondiffractive region as predicted by (22).

\section{CONCLUSION}

A new simple procedure to design scalar metasurfaces antennas capable of producing polarized beams in the far-field and near-field zones has been presented.

The theory has been first validated by the design of an LP and a CP antenna using a uniform a modulation index exited by a pin-like feeder. Then, a more sophisticated design capable of improving the aperture efficiency of the antenna has been presented. The novelty of this solution is the introduction of a directional feeder and the use of a nonuniform modulation index. Finally, a near-field application of metasurfaces has been presented for the generation of a Bessel beam launcher.

Measurements and full-wave results validate the proposed approach.

\section{APPENDIX}

This appendix presents a periodic GO approximation of the SW propagating over the modulated metasurface.

Decompose the wave vector in its transverse and normal components with respect to the metasurface: $\mathbf{k}=\mathbf{k}_{t}+k_{z} \hat{\mathbf{z}}$. A scalar potential describing a $\mathrm{TM}_{z} \mathrm{SW}$ can be written as $\Psi\left(\mathbf{r}^{\prime}\right)=\Psi_{t}\left(\rho^{\prime}\right) e^{-j k_{z} z}$, where the transverse scalar potential $\Psi_{t}$ should satisfy the 2-D wave equation

$$
\nabla_{t}^{2} \Psi_{t}\left(\rho^{\prime}\right)+k_{t}^{2}\left(\rho^{\prime}\right) \Psi_{t}\left(\rho^{\prime}\right)=0
$$

where $\nabla_{t}^{2}=\nabla^{2}-\partial^{2} / \partial z^{2}$, and the transverse wave number $k_{t}\left(\rho^{\prime}\right)$ depends on the position.

In optic approximation, $k_{t}$ is calculated using the local dispersion equation (2), then the normal wavenumber is obtained as $k_{z}=\left(k_{0}^{2}-k_{t}^{2}\right)^{1 / 2}$. However, this choice cannot describe the surface-to-LW transition since (2) gives rise only to real wavenumbers. This problem could be overcome by using the local periodic dispersion solution given by (5).

The obtained complex wavenumber $k_{t}\left(\boldsymbol{\rho}^{\prime}\right)$ can be written as

$$
k_{t}\left(\rho^{\prime}\right)=\bar{k}_{t} \eta_{\mathrm{eff}}\left(\boldsymbol{\rho}^{\prime}\right)
$$

where $\bar{k}_{t}$ is the wavenumber of an SW propagating over the average impedance $Z_{s}=j \bar{X}_{s}$, given by (2), and $\eta_{\mathrm{eff}}$ is a complex quantity. 
We are looking for a solution of (A1) of the form

$$
\Psi_{t}\left(\boldsymbol{\rho}^{\prime}\right)=\psi\left(\boldsymbol{\rho}^{\prime}\right) e^{-j \bar{k}_{t} S\left(\boldsymbol{\rho}^{\prime}\right)}
$$

where the unit vector orthogonal to the wavefront is given by the normalized gradient of the phase function

$$
\hat{\mathbf{k}}_{t}\left(\boldsymbol{\rho}^{\prime}\right)=\frac{\nabla_{t} S\left(\boldsymbol{\rho}^{\prime}\right)}{\left\|\nabla_{t} S\left(\boldsymbol{\rho}^{\prime}\right)\right\|} .
$$

Using (A3) in (A1) and equating real and imaginary parts of the equation leads to the following couple of equations:

$$
\begin{gathered}
\nabla_{t}^{2} \psi\left(\boldsymbol{\rho}^{\prime}\right)+\psi\left(\boldsymbol{\rho}^{\prime}\right) \bar{k}_{t}^{2}\left[\operatorname{Re}\left\{\eta_{\mathrm{eff}}^{2}\left(\boldsymbol{\rho}^{\prime}\right)\right\}-\nabla_{t} S\left(\boldsymbol{\rho}^{\prime}\right) \cdot \nabla_{t} S\left(\boldsymbol{\rho}^{\prime}\right)\right]=0 \\
2 \nabla_{t} \psi\left(\boldsymbol{\rho}^{\prime}\right) \cdot \nabla_{t} S\left(\boldsymbol{\rho}^{\prime}\right)+\psi\left(\boldsymbol{\rho}^{\prime}\right)\left[\nabla_{t}^{2} S\left(\boldsymbol{\rho}^{\prime}\right)-\bar{k}_{t} \operatorname{Im}\left\{\eta_{\mathrm{eff}}^{2}\left(\boldsymbol{\rho}^{\prime}\right)\right\}\right] \stackrel{\text { (A5) }}{=0 .}
\end{gathered}
$$

Using the GO approximation [29], $\nabla_{t}^{2}\left\{\psi\left(\boldsymbol{\rho}^{\prime}\right)\right\}$ is negligible in (A5) leading to the eikonal equation

$$
\nabla_{t} S\left(\rho^{\prime}\right) \cdot \nabla_{t} S\left(\rho^{\prime}\right)=\left|\nabla_{t} S\left(\boldsymbol{\rho}^{\prime}\right)\right|^{2}=\operatorname{Re}\left\{\eta_{\mathrm{eff}}^{2}\left(\boldsymbol{\rho}^{\prime}\right)\right\} .
$$

The impedance normalized modal vectors are defined as [30]

$$
\begin{aligned}
\mathbf{e}_{t}^{\mathrm{TM}}\left(\mathbf{r}^{\prime}\right) & =-\nabla_{t} \Psi_{t}\left(\mathbf{r}^{\prime}\right) \\
\mathbf{h}_{t}^{\mathrm{TM}}\left(\mathbf{r}^{\prime}\right) & =\hat{\mathbf{z}} \times \mathbf{e}_{t}^{\mathrm{TM}}\left(\mathbf{r}^{\prime}\right) .
\end{aligned}
$$

Under GO approximation $\left\|\nabla_{t} S\left(\boldsymbol{\rho}^{\prime}\right)\right\| \ll \bar{k}_{t}\left|\nabla_{t}\left\{S\left(\boldsymbol{\rho}^{\prime}\right)\right\}\right|$ $\psi\left(\rho^{\prime}\right)$, the electric field in (A8) can be written as

$$
\mathbf{e}_{t}^{\mathrm{TM}}\left(\boldsymbol{\rho}^{\prime}\right)=j \bar{k}_{t} \Psi\left(\boldsymbol{\rho}^{\prime}\right) \sqrt{\operatorname{Re}\left\{\eta_{\mathrm{eff}}^{2}\left(\boldsymbol{\rho}^{\prime}\right)\right\}} \hat{\mathbf{k}}_{t}\left(\boldsymbol{\rho}^{\prime}\right) .
$$

Introducing the following quantities:

$$
\begin{aligned}
A^{\mathrm{TM}}\left(\boldsymbol{\rho}^{\prime}\right) & =j \bar{k}_{t} \psi\left(\boldsymbol{\rho}^{\prime}\right) \sqrt{\operatorname{Re}\left\{\eta_{\mathrm{eff}}^{2}\left(\boldsymbol{\rho}^{\prime}\right)\right\}} \\
\mathbf{k}_{\mathbf{t}}\left(\boldsymbol{\rho}^{\prime}\right) \cdot \boldsymbol{\rho}^{\prime} & =\bar{k}_{t} \eta_{\mathrm{eff}}\left(\boldsymbol{\rho}^{\prime}\right) S\left(\boldsymbol{\rho}^{\prime}\right) \\
\hat{\mathbf{e}}_{t}^{\mathrm{TM}}\left(\boldsymbol{\rho}^{\prime}\right) & =\hat{\mathbf{k}}_{t}\left(\boldsymbol{\rho}^{\prime}\right) \\
\hat{\mathbf{h}}\left(\boldsymbol{\rho}^{\prime}\right) & =\hat{\mathbf{z}} \times \hat{\mathbf{e}}_{t}^{\mathrm{TM}}\left(\boldsymbol{\rho}^{\prime}\right)
\end{aligned}
$$

the normalized wave vectors on the surface $(z=0)$ can be written as in Section II-A

$$
\left\{\begin{array}{l}
\mathbf{h}_{t}^{\mathrm{TM}}\left(\boldsymbol{\rho}^{\prime}\right)=A^{\mathrm{TM}}\left(\boldsymbol{\rho}^{\prime}\right) e^{-j \mathbf{k}_{t}\left(\boldsymbol{\rho}^{\prime}\right) \cdot \boldsymbol{\rho}^{\prime}} \hat{\mathbf{h}}_{t}^{\mathrm{TM}}\left(\boldsymbol{\rho}^{\prime}\right) \\
\mathbf{e}_{t}^{\mathrm{TM}}\left(\boldsymbol{\rho}^{\prime}\right)=A^{\mathrm{TM}}\left(\boldsymbol{\rho}^{\prime}\right) e^{-j \mathbf{k}_{t}\left(\boldsymbol{\rho}^{\prime}\right) \cdot \boldsymbol{\rho}^{\prime}} \hat{\mathbf{e}}_{t}^{\mathrm{TM}}\left(\boldsymbol{\rho}^{\prime}\right) .
\end{array}\right.
$$

Finally, the electromagnetic mode propagating over the modulated metasurface is obtained by choosing the surface impedance as transmission line impedance $\left[H\left(\rho^{\prime}\right)=\mathbf{h}_{t}^{\mathrm{TM}}\left(\boldsymbol{\rho}^{\prime}\right)\right.$, $\left.E\left(\rho^{\prime}\right)=Z_{s} \mathbf{h}_{t}^{\mathrm{TM}}\left(\boldsymbol{\rho}^{\prime}\right)\right]$.

\section{ACKNOWLEDGMENT}

The authors would like to thank the anonymous reviewers for the useful comments, which have improved the quality of this paper.

\section{REFERENCES}

[1] C. L. Holloway, E. F. Kuester, J. A. Gordon, J. O'Hara, J. Booth, and D. R. Smith, "An overview of the theory and applications of metasurfaces: The two-dimensional equivalents of metamaterials," IEEE Trans. Antennas Propag., vol. 54, no. 2, pp. 10-35, Apr. 2012.

[2] B. H. Fong, J. S. Colburn, J. J. Ottusch, J. L. Visher, and D. F. Sievenpiper, "Scalar and tensor holographic artificial impedance surfaces," IEEE Trans. Antennas Propag., vol. 58, no. 10, pp. 3212-3221, Oct. 2010.
[3] G. Minatti, F. Caminita, M. Casaletti, and S. Maci, "Spiral leaky-wave antennas based on modulated surface impedance," IEEE Trans. Antennas Propag., vol. 59, no. 12, pp. 4436-4444, Dec. 2011.

[4] A. M. Patel and A. Grbic, "A printed leaky-wave antenna based on a sinusoidally-modulated reactance surface," IEEE Trans. Antennas Propag., vol. 59, no. 6, pp. 2087-2096, Jun. 2011.

[5] D. J. Gregoire, "Impedance modulation patterns for artificial-impedancesurface antennas," in Proc. IEEE Antennas Propag. Soc. Int. Symp., Orlando, FL, USA, Jul. 2013, pp. 2339-2340.

[6] C. Pfeiffer and A. Grbic, "A printed, broadband Luneburg lens antenna," IEEE Trans. Antennas Propag., vol. 58, no. 9, pp. 3055-3059, Sep. 2010.

[7] M. Bosiljevac, M. Casaletti, F. Caminita, Z. Sipus, and S. Maci, "Non-uniform metasurface Luneburg lens antenna design," IEEE Trans. Antennas Propag., vol. 60, no. 9, pp. 4065-4073, Sep. 2012.

[8] M. Huang, S. Yang, F. Gao, R. Quarfoth, and D. Sievenpiper, "A 2-D multibeam half Maxwell fish-eye lens antenna using high impedance surfaces," IEEE Antennas Wireless Propag. Lett., vol. 13, pp. 365-368, 2014.

[9] K. Hannam, D. A. Powell, I. V. Shadrivov, and Y. S. Kivshar, "Broadband chiral metamaterials with large optical activity," Phys. Rev. B, vol. 89, p. 125105, Mar. 2014.

[10] C. Pfeiffer and A. Grbic, "Controlling vector Bessel beams with metasurfaces," Phys. Rev. Appl., vol. 2, no. 4, p. 044012, 2014.

[11] T. Dong, X. Ma, and R. Mittra, "Modeling large nonuniform optical antenna arrays for metasurface application," J. Appl. Phys., vol. 114, no. 4, pp. 043103-1-043103-10, Jul. 2013.

[12] X. Wan, W. X. Jiang, H. F. Ma, and T. J. Cui, "A broadband transformation-optics metasurface lens," Appl. Phys. Lett., vol. 104 no. 15, pp. 151601-1-151601-4, 2014.

[13] E. Martini and S. Maci, "Metasurface transformation theory," in Transformation Electromagnetics and Metamaterials: Fundamental Principles and Applications, D. H. Werner and D.-H. Kwon, Eds. Berlin, Germany: Springer, 2013.

[14] N. Engheta, "Metamaterials: where do we go from here?" in Proc. Metamaterials 7th Int. Congr. Adv. Electromagn. Mater. Microwaves Optics, Bordeaux, France, Sep. 2013, pp. 16-19.

[15] A. Oliner and A. Hessel, "Guided waves on sinusoidally-modulated reactance surfaces," IRE Trans. Antennas Propag., vol. 7, no. 5, pp. 201-208, Dec. 1959.

[16] S. Maci, G. Minatti, M. Casaletti, and M. Bosiljevac, "Metasurfing: Addressing waves on impenetrable metasurfaces," IEEE Antennas Wireless Propag. Lett., vol. 10, pp. 1499-1502, 2011.

[17] G. Minatti, S. Maci, P. De Vita, A. Freni, and M. Sabbadini, "A circularly-polarized isoflux antenna based on anisotropic metasurface," IEEE Trans. Antennas Propag., vol. 60, no. 11, pp. 4998-5009, Nov. 2012.

[18] R. Quarfoth and D. Sievenpiper, "Broadband unit-cell design for highly anisotropic impedance surfaces," IEEE Trans. Antennas Propag., vol. 62, no. 8, pp. 4143-4152, Aug. 2014.

[19] D. M. Pozar, Microwave Engineering, 4th ed. New York, NY, USA: Wiley, 2011.

[20] M. Smierzchalski, M. Casaletti, M. Ettorre, R. Sauleau, and N. Capet, "Scalar metasurface antennas with tilted beam," in Proc. EuCAP, Lisbon, Portugal, Apr. 2015, pp. 1-3.

[21] N. Capet, M. Casaletti, R. Sauleau, M. Ettorre, and M. Smierzchalski, "Dispositif à métasurface d'impédance modulée et variable pour l'émission/réception d'ondes électromagnétiques," French Patent, 2014.

[22] S. K. Podilchak, A. P. Freundorfer, and Y. M. M. Antar, "A new leaky-wave antenna design using simple surface-wave power routing techniques," in Proc. IEEE APSURSI, Jul. 2011, pp. 3052-3054.

[23] M. Ettorre, S. Bruni, G. Gerini, A. Neto, N. Llombart, and S. Maci, "Sector PCS-EBG antenna for low-cost high-directivity applications," IEEE Antennas Wireless Propag. Lett., vol. 6, pp. 537-539, 2007.

[24] D. Mcgloin and K. Dholakia, "Bessel beams: Diffraction in a new light," Contemp. Phys., vol. 46, no. 1, pp. 15-28, Jan. 2005.

[25] M. Ettorre and A. Grbic, "Generation of propagating Bessel beams using leaky-wave modes," IEEE Trans. Antennas Propag., vol. 60, no. 8, pp. 3605-3613, Aug. 2012.

[26] M. Ettorre et al., "On the near-field shaping and focusing capability of a radial line slot array," IEEE Trans. Antennas Propag., vol. 62, no. 4, pp. 1991-1999, Apr. 2014.

[27] M. Albani, S. C. Pavone, M. Casaletti, and M. Ettorre, "Generation of non-diffractive Bessel beams by inward cylindrical traveling wave aperture distributions," Opt. Exp., vol. 22, no. 15, pp. 18354-18364, 2014. 
[28] M. Ettorre, S. C. Pavone, M. Casaletti, and M. Albani, "Experimental validation of Bessel beam generation using an inward Hankel aperture distribution," IEEE Trans. Antennas Propag., vol. 63, no. 6, pp. 2539-2544, Jun. 2015.

[29] L. B. Felsen and N. Marcuvitz, Radiation and Scattering of Waves. Upper Saddle River, NJ, USA: Prentice-Hall, 1973.

[30] N. Marcuvitz, Waveguide Handbook. Stevenage, U.K.: Peregrinus, 1986. 